\title{
Image Pattern Recognition in Natural Environment Using Morphological Feature Extraction
}

\author{
Yonggwan Won, Jiseung Nam, and Bae-Ho Lee \\ Department of Computer Engineering \\ Chonnam National University \\ 300 Yongbong-Dong Puk-Gu Kwangju, Korea
}

\begin{abstract}
The gray-scale morphological Hit-or-Miss transform is theoretically invariant to vertical translation of the input function, which is analogous to gray-value shift of the input images. Designing optimal structuring elements for the Hit-or-Miss transform operator is achieved by neural network learning methodology using a shared-weight neural network (SWNN) architecture. Early stage of the neural network system performs feature extraction using the operator, while the late stage does classification. In experimental studies, this morphological feature-based neural network (MFNN) system is applied to location of human face and automatic recognition of vehicle license plate to examine the property of the operator. The results of the experimental studies show that the gray-scale morphological Hit-or-Miss transform operator is reducing the effects of lighting variation.
\end{abstract}

\section{Introduction}

Due to lighting and illumination variability, the same object or pattern can be recognized differently [1]. Even a slight change in lighting environment or shading across the object region can easily influence the classification results. This fact makes recognition of objects or patterns difficult. While this issue has been a great concern in computer vision, most efforts have been devoted to dealing with some property or features of the image, i.e., edge or distribution, and reflectance property of the object surface [2]. Unfortunately, those approaches are feasible for fully automated image recognition and analysis.

Recognition methods using low-dimensional representation of image objects have been recently introduced [3], [4], which often termed appearance-based method differed from the feature-based methods. The methods demonstrated ease of implementation and accuracy. However, they perform recognition reliably provided that the object or pattern has been previously seen under similar circumstances. This drawback limits the methods in application for a problem in lighting variability.

In this paper, we propose an "operation-based" approach to resolve the problem of lighting variation. We first introduce an operator, gray-scale morphological Hit-orMiss transform, which is theoretically insensitive to lighting variation in image acquisition environment. This operator is applied to the nodes in the feature extraction stage of a shared-weight neural network (SWNN) [5]. This morphological feature- 
based neural network (MFNN) system is then applied to location of human face and recognition of vehicle license plate of which primary goal is to recognize four consecutive numeric numbers in the license plate. Images were collected in various lighting conditions and at different locations. The experimental results demonstrate that the gray-scale morphological Hit-or-Miss transform operator is well reducing the effects caused by the illumination change in image acquisition environment.

\section{Morphological Operations for Gray-Scale Images}

In this section, we first describe some preliminary definitions and notations which are counterparts for those of binary morphology [6]. Then, we introduce two essential gray-scale morphological operations, erosion and dilation, and Hit-or-Miss transform that plays a role of feature extraction for the morphological filter neural network system.

\subsection{Definitions and Notations}

There are three translations defined as :

- translation : horizontal shift to the right by $\mathbf{z}$

$$
f_{\mathbf{z}}(\mathbf{x})=f_{\mathbf{z}}(\mathbf{x}-\mathbf{z})
$$

- offset : vertical translation by the amount $y$

$$
(f+y)(\mathbf{x})=f(\mathbf{x})+y
$$

- morphological translation : translation and offset

$$
\left(f_{\mathbf{z}}+y\right)(\mathbf{x})=f(\mathbf{x}-\mathbf{z})+y
$$

The function $g$ is beneath the function $f$, denoted by $g<<f$, if $D[g] \subseteq D[f]$ and $g(\mathbf{x}) \leq f(\mathbf{x})$ for $\mathbf{x} \in D[g]$ where $D[g]$ denotes the domain of the function $g$.

Counterparts to intersection and union in binary morphology are minimum and maximum, respectively. By allowing the negative infinity value for $f$ and $g$ if $\mathbf{x}$ is not in the domain, the minimum is defined by

$$
(f \wedge g)(\mathbf{x})=\min \{f(\mathbf{x}), g(\mathbf{x})\} .
$$

In similar way, the maximum is defined by

$$
(f \wedge g)(\mathbf{x})=\left\{\begin{array}{cc}
f(\mathbf{x}) & \text { if } \mathbf{x} \in D[f] \text { and } \mathbf{x} \notin D[g] \\
\max \{f(\mathbf{x}), g(\mathbf{x})\} & \text { if } \mathbf{x} \in\{D[f] \cap D[g]\} \\
g(\mathbf{x}) & \text { if } \mathbf{x} \notin D[f] \text { and } \mathbf{x} \in D[g] \\
\text { undefined } & \text { if } \mathbf{x} \in\{D[f] \cap D[g]\}
\end{array}\right.
$$


The gray-scale operation analog to rotation of a set about its origin is reflection. The reflection of a function $h$ is defined by

$$
h^{*}(\mathbf{x})=-h(-\mathbf{x}) \text {. }
$$

\subsection{Erosion and Dilation}

Using notion of "fitting", two essential gray-scale morphological operations erosion and dilation are defined [6], [7]. The gray-scale erosion of a function $f$ by another function (called structuring element) $g$ is defined by

$$
(f \theta g)(\mathbf{x})=\max \left\{y: g_{\mathbf{x}}+y<<f\right\} .
$$

Instead of finding the maximum "offset", we can find the "minimum difference" between the function $f(\mathbf{z})$ 's and the structuring element $g(\mathbf{z})$ 's for all $\mathbf{z} \in D\left[g_{\mathbf{x}}\right]$, which results in

$$
(f \theta g)(\mathbf{x})=\min \left\{f(\mathbf{z})-g_{\mathbf{x}}(\mathbf{z}): \mathbf{z} \in D\left[g_{\mathbf{x}}\right]\right\} .
$$

Note that this measures how well the shape of the structuring element $g$ fits under the function $f$, and it is only defined at any point where $g_{\mathbf{x}}<<f$.

The gray-scale dilation of a function $f$ by a structuring element $g$ can be defined in a dual manner to the gray-scale erosion. This notion leads to the following definitions:

$$
(f \oplus g)(\mathbf{x})=\min \left\{y:(g)_{\mathbf{x}}+y>>f\right\}
$$

and

$$
(f \oplus g)(\mathbf{x})=\max \left\{f(\mathbf{z})-(g *)_{\mathbf{x}}(\mathbf{z}): \mathbf{z} \in D\left[(g *)_{\mathbf{x}}\right]\right\} .
$$

The gray-scale dilation indirectly measures how well the shape of the structuring element $g$ fits above the function $f$.

Several algebraic properties of the gray-scale erosion and dilation are described well in [6], [7]. From Eq. (7) to Eq. (10), note that the outputs of erosion and dilation are not invariant to offset (vertical translation) of the function $f$.

\subsection{Hit-or-Miss Transform}

In binary morphology, the Hit-or-Miss transform probes the inside and the outside of the image $\boldsymbol{A}$ (i.e., set) with a pair of disjoint structuring elements $\boldsymbol{B}=(\boldsymbol{E}, \boldsymbol{F})$. Mathematical formulation of this transform is defined by

$$
\boldsymbol{A} \otimes \boldsymbol{B}=(\boldsymbol{A} \theta \boldsymbol{E})-\left(\boldsymbol{A}^{c} \oplus \boldsymbol{F}\right) .
$$

Using the notion of "umbra transform" which is a way to consider a gray-scale image a binary image (i.e., a set) in 3-dimensional Euclidean space [6], a novel gray-scale Hit-or-Miss transform is defined by 


$$
\begin{aligned}
f \otimes(h, m) & =(f \theta h)-(f \oplus m) \\
& =\min \left\{f(\mathbf{z})-g_{\mathbf{x}}(\mathbf{z})\right\}-\max \left\{f(\mathbf{z})-\left(g^{*}\right)_{\mathbf{X}}(\mathbf{z})\right\}
\end{aligned}
$$

where $\mathbf{z} \in D\left[g_{\mathbf{x}}\right]$ and $D\left[g_{\mathbf{x}}\right]=D\left[\left(\mathrm{~g}^{*}\right)_{\mathbf{x}}\right]$. Complete procedure to achieve Eq. (12) is provided in [8].

The gray-scale Hit-or-Miss transform has a useful property: if $\lambda \in \mathbf{R}$, then, $f \otimes(h, m)=(f+\lambda) \otimes(h, m)$. Thus, the gray-scale Hit-or-Miss transform can reduce effects caused by illumination and sensor parameter changes for image acquisition systems. Proof of this property is provided in Appendix.

\section{Morphological Filter Neural Network}

\subsection{Architecture of Neural Network System}

Shared-weight neural network (SWNN) is composed of two cascade sub-networks: a feature extraction network followed by a classification network [5]. The feature extraction network usually has two-dimensional array for the input image and performs convolutional operations over its input with the weight kernels, which generates feature map. The weight kernel, two-dimensional local connection, is identical for the nodes in the same feature map. Each node is corresponding to certain position in the input. There can be more than one feature map layer and each layer can have more than one feature map. The classification network is an ordinary feedforward network of which the input is the feature maps in the last feature extraction layer. A more precise description of the SWNN is given in [8].

The SWNN is trained with the inputs that have fixed size. The output indicates a class to which the input belongs. For locating an object in an input image of arbitrary size, one should scan entire image with the SWNN. An extended shared-weight neural network (ESWNN) architecture that probes entire image more efficiently with the weight kernels obtained by the SWNN is proposed in [8], [9], called scanning mode operation. Result of scanning mode operation is a detection plane which presents the possibility of object existence at each point of the input image.

Our work is mainly focused on locating and recognizing image patterns (human faces and numeric numbers) simultaneously in the input image scenes (indoor images and vehicle images). For this purpose, the SWNN and ESWNN provide the suitable architecture.

\subsection{Feature Extraction Operation}

Morphological processing has been widely used for pattern recognition as a feature extraction methodology [6], [10]. The outcome of the morphological processing is highly dependent on the characteristics of the structuring elements [11]. In terms of the mathematical morphology, the weight kernel of the SWNN can be considered a structuring element. 
The nodes in the feature extraction network of the ordinary SWNN perform the usual linear weighed sum followed by mapping through sigmoid function. Our MFNN performs gray-scale Hit-or-Miss transform defined in Eq. (12). To perform this operation, each node should have two structuring elements: one for erosion and the other for dilation. Output of the node is defined by

$$
a(x, y)=\operatorname{net}_{(c, d)}^{h}-\operatorname{net}_{(i, j)}^{m}
$$

where

$$
\operatorname{net}_{(c, d)}^{h}=\min _{(c, d) \in D\left[h_{(x, y)}\right.}\left\{a(c, d)-h_{(x, y)}(c, d)\right\}
$$

and

$$
\operatorname{net}_{(i, j)}^{m}=\max _{(i, j) \in D\left[m_{(x, y)]}\right.}\left\{a(i, j)-m_{(x, y)}(i, j)\right\} .
$$

In Eq. (13), (14) and (15), $\mathrm{D}\left[h_{(x, y)}\right]=\mathrm{D}\left[m_{(x, y)}\right]$, and $(x, y)$ indicates the location of the output node in the feature map, while $(c, d)$ and $(i, j)$ do those of the input node. Also, $h_{(x, y)}$ and $m_{(x, y)}$ represent the structuring elements for erosion and dilation, respectively.

\subsection{Learning Rules}

Since the learning rules for the nodes in the classification network is widely available in neural network literature [12], we only provide those for the feature extraction network.

For each node in the feature extraction network, the learning rules for each structuring element should be provided. Based on the gradient descent method with application of chain rule, the learning rules for the feature extraction network are summarized as [8]:

$$
\Delta h_{(x, y)}(c, d)=\eta \delta_{(x, y)} \frac{\partial \operatorname{net}_{(x, y)}^{h}}{\partial h_{(x, y)}(c, d)}
$$

and

$$
\Delta m_{(x, y)}(i, j)=-\eta \delta_{(x, y)} \frac{\partial \operatorname{net}_{(x, y)}^{m}}{\partial m_{(x, y)}(i, j)}
$$

where

$$
\delta_{(x, y)}=\sum_{k} \delta_{k} w_{k}(x, y)
$$

for the nodes in the last feature extraction layer and

$$
\delta_{(x, y)}=\sum_{(p, q)} \delta_{(p, q)}\left(\frac{\partial \text { net }_{(p, q)}^{h}}{\partial a(x, y)}-\frac{\partial \text { net }_{(p, q)}^{m}}{\partial a(x, y)}\right)
$$

for the nodes in the hidden layers. In Eq. (18), $k$ indexes the nodes in the first hidden layer of the feedforward classification network.

Assume for a while that all derivatives exist. Then, they are given by 


$$
\begin{gathered}
\frac{\partial \text { net }_{(x, y)}^{h}}{\partial h_{(x, y)}(c, d)}=\left\{\begin{array}{cc}
-1 & \text { if }(c, d)=\underset{(s, t) \in D\left[h_{(x, y)}\right]}{\arg \min }\left\{a(s, t)-h_{(x, y)}(s, t)\right\} \\
0 & \text { otherwise }
\end{array}\right\} \\
\frac{\partial \operatorname{net}_{(x, y)}^{m}}{\partial m_{(x, y)}(c, d)}=\left\{\begin{array}{rr}
-1 & \text { if }(c, d)=\underset{(s, t) \in D\left[m_{(x, y)}\right]}{\arg \min }\left\{a(s, t)-m_{(x, y)}(s, t)\right\} \\
0 & \text { otherwise }
\end{array}\right. \\
\frac{\partial \operatorname{net}_{(p, q)}^{h}}{\partial a(x, y)}= \begin{cases}1 & \text { if }(x, y)=\underset{(s, t) \in D\left[h_{(p, q)}\right]}{\arg \min }\left\{a(s, t)-h_{(p, q)}(s, t)\right\} \\
0 & \text { otherwise }\end{cases} \\
\frac{\partial \operatorname{net}_{(p, q)}^{m}}{\partial a(x, y)}= \begin{cases}1 & \text { if }(x, y)=\underset{(s, t) \in D\left[m_{(p, q)}\right]}{\arg \min }\left\{a(s, t)-m_{(p, q)}(s, t)\right\} \\
0 & \text { otherwise }\end{cases}
\end{gathered}
$$

Note that erosion and dilation (i.e., net ${ }^{h}$ and net ${ }^{m}$ ) are only piecewise differentiable. Mathematically, the derivative is not defined for the case that there are more than one index for $(c, d)$ and $(i, j)$ in Eq. (14) and (15). In this case, we simply arbitrarily select one index.

\section{Image Data Sets}

\subsection{Face Images}

Hundreds of human face images were collected from variety of resources. We first arbitrarily selected 1000 images that only include frontal view of human face with minimum background. The collected images were then resized into 132x100. This data set was used only for training the MFNN.
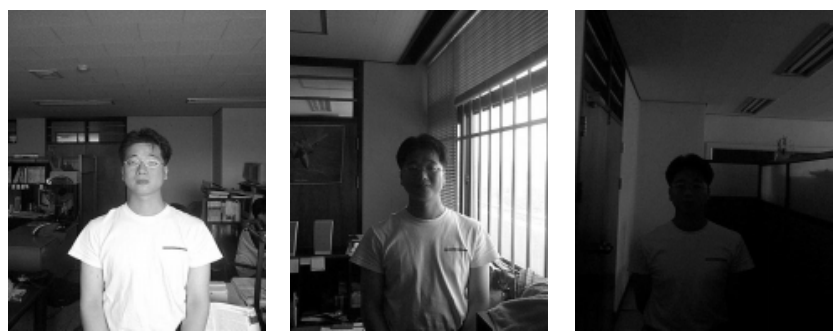

Fig. 1. Some examples of indoor images (test images)

\subsection{Indoor Images}

We first collected 640x480 gray-scale images from the offices in the university under the appropriate lighting condition. From this data set, three thousands of $132 \times 100$ sub-images were collected. This sub-image data set was used as background (nonface) to train the MFNN together with the images described in the section 4.1. 
Twenty images of $640 \times 480$ were also independently collected. They include human faces of which the size was approximately $132 \times 100$. This data set was used only to test the MFNN. Some images of the test data set are presented in Fig. 1.

\subsection{Vehicle Images}

The vehicle images were collected from image databases of speed control systems. The system captured the images of vehicles approximately at the 100-meter distance. The size of the images is $480 \times 512$. From the database, we selected 1000 images in reasonably good condition to collect the training images described in the following section 4.4. Some of example images of the training set are presented in Fig 2.

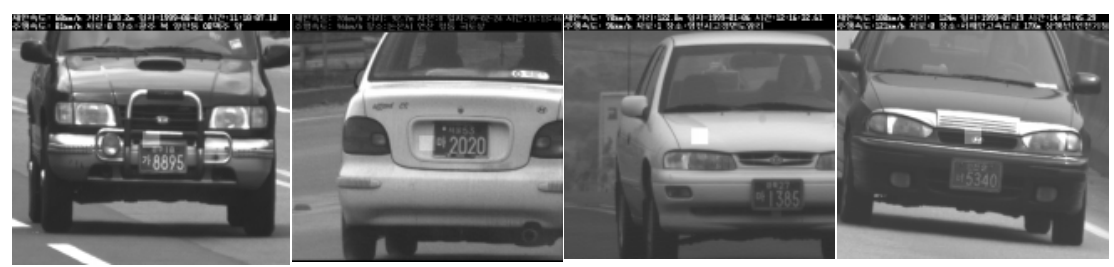

Fig. 2. Some example images of the training set

\subsection{License Number Images}

From the training image set described in the section 4.3 , we manually collected subimages of digits in the license plate, of which the size is $35 \times 19$. From those subimages, we arbitrarily selected 200 image patterns for each digit. This data set was only used for training the network along with the non-digit image set described in the section 4.5. Some examples of digit image along with the images of the license plate are shown in Fig 3. Note that we only considered the four consecutive digits.
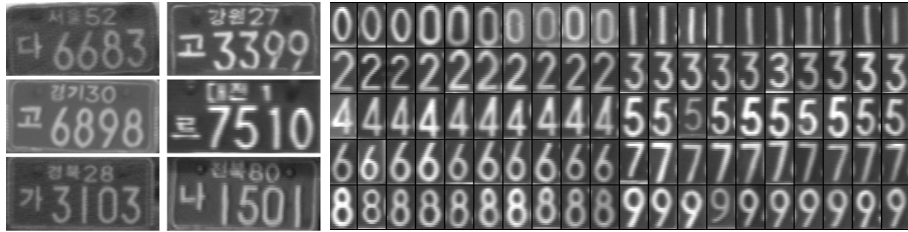

Fig. 3. Some examples of license plates and digits

\subsection{Non-digit Images}

Non-digit sub-images were collected randomly during the training process from the outside of the digit region. A bounding box that had a size of $23 \times 11$ and was centered at the center of a digit defined the digit region. This method can overcome problems caused by wide variation of background (non-digit). 


\section{Human Face Detection}

In this experimental study, we trained the MFNN with the sub-image sets described in the section 4.1 and 4.2. During training the network, each image was presented by shifting three pixels in both horizontal and vertical directions around the center of the image. Background region was filled with the image region discarded by shift

In this experiment, the feature extraction network had a single layer with two feature maps. The size of the structuring elements was $5 \times 5$. The feature extraction operation was done at every other point, which dramatically reduces computational complexity [8], [9]. Therefore, the output of the feature extraction operation had the half size of the input. The classification network also had a single hidden layer with four nodes and two output nodes: one represents the human face while the other does the background.

To locate the human faces using the ESWNN architecture, we used the detection plane generated by the output node representing the human face class. The detection plane was first multiplied by 255 and thresholded by the value 230 . We then repeatedly applied the erosion with $3 \times 3$ flat structuring element until the last nonempty eroded image was obtained. The MFNN located the human faces in 16 images out of 20 test images with some false alarms that classified the background a human face. The undetected ones were captured in very dark environments such as the last image in Fig. 1. In comparison, the ordinary SWNN only detected the faces in 12 images and produced many false alarms.

\section{Vehicle Identification}

We trained the MFNN with the image data sets described in the section 4.4 and 4.5. The feature extraction network had a single layer with 5 feature maps. The size of the structuring elements was $5 \times 5$. The classification network had also a single hidden layer with 25 nodes and 10 output nodes: each output node corresponded to a number from 0 to 9 .

Training was stopped when the number of epochs reached 200 or the RMSSE was below 0.05 . When a non-digit image was presented, desired output values for all output nodes were 0 . Learning rate 0.05 and the momentum 0.8 were used. Trained network was then test with the image data set described in the section 4.3 using the ESWNN architecture.

We first thresholded 10 detection planes with 0.87 to generate binary detection planes, and then performed pixel-by-pixel logical OR operation by

$$
\boldsymbol{D}(x, y)=\bigcup_{i=0}^{9} \boldsymbol{P}_{i}(x, y)
$$

where $\boldsymbol{P}_{i}$ indicates a detection plane. Fig. 4 shows test images and their corresponding binary images $\boldsymbol{D}$ called detection image plane. The white pixel in the $\boldsymbol{D}$ image was then labeled with the number to which it corresponds.

We first extracted coordinates of 1's from the detection image plane $\boldsymbol{D}$. We used a coordinate system that had the origin at the left-top corner and $x$ as the horizontal axis and $y$ as the vertical axis. Assume that the pixel $\left(x_{i}, y_{i}\right)$ is labeled in $p$ and $\left(x_{i+1}, y_{i+1}\right)$ is 
labeled in $q$. Then, a simple rule " $q$ is the next digit to $p$ if $\left(x_{i}+15<x_{i+1}<x_{i}+22\right) \cap\left(y_{i}-4<\right.$ $\left.y_{i+1}<y_{i}+4\right)$ " can determine four consecutive numbers. This rule was generated based on the facts observed from the detection planes.
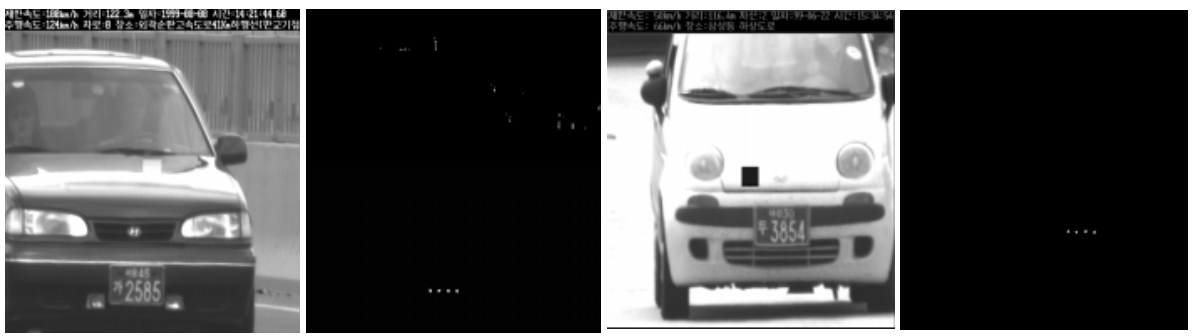

Fig. 4. Examples of test results

It is possible that a white pixel have multiple labels. In this case, we simply selected the label corresponding to the detection plane that had larger value for that pixel. Test on arbitrarily selected 500 images resulted in 441 corrects $(88.2 \%)$. Only 6 vehicles $(1.2 \%)$ were miss-identified. 53 vehicles $(10.6 \%)$ were rejected. The border of the plate frame, which is easily confused as the digit 1 and thus generated five consecutive numbers, caused most of rejections.

\section{Conclusion}

Lighting and illumination change makes recognition of objects difficult. We proposed a gray-scale morphological Hit-or-Miss transform which has a useful property that can reduce effects due to lighting condition and sensor parameter changes for image acquisition systems. The gray-scale morphological Hit-or-Miss transform is applied to node operation of the feature extraction network in the shared-weight network.

We examined the property of the operator by performing two experimental studies: human face detection and vehicle identification. Results of our experiments demonstrate that the MFNN is immune to reasonable lighting changes.

\section{Acknowledgement}

This work was supported by Korea Research Foundation Grant. (KRF-99-041)

\section{References}

1. Horn, B. K. P.: Robot Vision. MIT Press and McGraw-Hill Book Company (1986)

2. Richter, R.: Correction of atmospheric and topographic effects for high resolution satellite imagery. Porc. Of the SPIE, Algorithms for Multispectral and Hyperspectral Imagery III. (1997) 216-224 
3. Hallinan, P.: A Low-dimensional representation of human faces for arbitrary lighting conditions. Proc. IEEE Conf. On Computer Vision and Pattern Recognition. (1994) 995999

4. Murase, H., Nayar, S.: Visual learning and recognition of 3-D Objects from appearance. Int. J. Computer Vision, Vol. 14. (1995) 5-24

5. le Cun, Y., Jackel, L. D., Boser, B., Denker, J. S., Graf, H. P., Guyon, I., Henderson, D., Howard, R. E., Hubbard, W.: Handwritten Digit Recognition: Application of Neural Network Chips and Automatic Learning. IEEE Communications Magazine. (1989) 41-64

6. Dougherty, E.: An Introduction to Morphological Image Processing. SPIE Optical Engineering Press (1992)

7. Serra, J.: Image Analysis and Mathematical Morphology, Vol. 1: Theoretical Advances. Academic Press, New York (1982)

8. Won, Y.: Nonlinear Correlation Filter and Morphology Neural Networks for Image Pattern and Automatic Target Recognition.. Ph.D. Dissertation, University of Missouri-Columbia (1995)

9. Gader, P., Miramonti, J., Won, Y., Coffield, P.: Segmentation-free Neural Network for Automatic Vehicle Detection. Neural Networks, Vol. 8. (1995) 1457-1473

10. Maragos, P.: A Representation theory for Morphological Image and Signal Processing. IEEE Trans. Pattern Analysis and Machine Intelligence, Vol. 11. (1989) 586-599

11. Dougherty, E., Loce, R.: Optimal Mean-Absolute Hit-or-Miss Filters. Optical Engineering, Vol. 32. (1993) 815-827

12. Rumelhart, D.E., Hinton, G.E., Williams, R.J.: Learning Internal Representations by Error Propagation. in Parallel Distributed Processing: Explorations in the Microstructure of Cognition. MIT Press, Cambridge (1986)

\section{Appendix: Proof for Property}

Let $\mathbf{z}$ be a point in $D[f]$, the domain of $f$. Assume that $D[h]=D[m]=\mathrm{D}\left[h^{*}\right]=D\left[m^{*}\right]$. Let $\mathrm{I}=D\left[h_{\mathrm{z}}\right]$. Then,

$$
\begin{aligned}
& {[f \otimes(h, m)](\mathbf{z})=\min _{\mathbf{x} \in \mathrm{I}}\left\{f(\mathbf{x})+\lambda-h_{\mathbf{z}}(\mathbf{x})\right\}-\underset{\mathbf{x} \in \mathrm{I}}{-\max _{1}}\left\{f(\mathbf{x})+\lambda-m_{\mathbf{z}}^{*}(\mathbf{x})\right\}} \\
& =\min _{\mathbf{x} \in \mathrm{I}}\left\{f(\mathbf{x})-h_{\mathbf{z}}(\mathbf{x})\right\}+\lambda-\max _{\mathbf{x} \in \mathrm{I}}\left\{f(\mathbf{x})-m_{\mathbf{z}}^{*}(\mathbf{x})\right\}-\lambda \\
& =\min _{\mathbf{x} \in \mathrm{I}}\left\{f(\mathbf{x})-h_{\mathbf{z}}(\mathbf{x})\right\}-\max _{\mathbf{x} \in \mathrm{I}}\left\{f(\mathbf{x})-m_{\mathbf{z}}^{*}(\mathbf{x})\right\} \\
& =[f \otimes(h, m)](\mathbf{z}) \text { Q.E.D. }
\end{aligned}
$$

